\title{
CROSS VALIDATION ON THE EQUALITY OF UAV-BASED AND CONTOUR-BASED DEMS
}

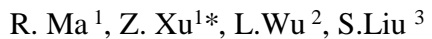 \\ ${ }^{1}$ College of Geoscience and Surveying Engineering, China University of Mining \& Technology, 100083 Beijing, China - \\ r.ma@student.cumtb.edu.cn; z.xu@cumtb.edu.cn \\ ${ }^{2}$ School of Geosciences and Info-physics, Central South University, 410006 Changsha, China - awulixin@263.net \\ ${ }^{3}$ Faculty of Geographical Science, Beijing Normal University, 100875 Beijing, China- liush@bnu.edu.cn
}

Commission III, ICWG III/IVb

KEY WORDS: UAV Photogrammetry, Digital Elevation Model, Topographic surveying, Ground Control Points, Accuracy analysis

\begin{abstract}
:
Unmanned Aerial Vehicles (UAV) have been widely used for Digital Elevation Model (DEM) generation in geographic applications. This paper proposes a novel framework of generating DEM from UAV images. It starts with the generation of the point clouds by image matching, where the flight control data are used as reference for searching for the corresponding images, leading to a significant time saving. Besides, a set of ground control points (GCP) obtained from field surveying are used to transform the point clouds to the user's coordinate system. Following that, we use a multi-feature based supervised classification method for discriminating non-ground points from ground ones. In the end, we generate DEM by constructing triangular irregular networks and rasterization. The experiments are conducted in the east of Jilin province in China, which has been suffered from soil erosion for several years. The quality of UAV based DEM (UAV-DEM) is compared with that generated from contour interpolation (ContourDEM). The comparison shows a higher resolution, as well as higher accuracy of UAV-DEMs, which contains more geographic information. In addition, the RMSE errors of the UAV-DEMs generated from point clouds with and without GCPs are $\pm 0.5 \mathrm{~m}$ and $\pm 20 \mathrm{~m}$, respectively.
\end{abstract}

\section{INTRODUCTION}

Digital elevation model (DEM) plays an important roles in soil conservation (Rao et al., 2014), ecological restoration (Liang et al., 2015), mining (Maxwell and Warner, 2015), urban planning (Shi and Yu, 2014), and geological disaster monitoring (Demirkesen, 2012). From a generative perspective, the methods of DEM generation are mainly divided into three categories: field surveying (Jones et al., 2012), aerial photogrammetry (Uysal et al., 2015) and satellite stereo imaging (Mukherjee et al., 2013). Comparing with the later method, the former two methods enable to achieve DEMs with high resolution, thus have been widely used for small area. Moreover, the development of advanced surveying instrument and aerial platforms, i.e., UAVs with the advantages of lowcost and time conservation, has made it easier to obtain DEM of terrain with different surface coverage. Many studies of DEMs generation with both UAV photogrammetry (De Souza et al., 2017; Rock et al., 2011) and contour interpolation (Ardiansyah and Yokoyama, 2002; Arun, 2013) can be found in literature and accuracy assessment of the achieved DEMs has been comprehensively investigated. The accuracy assessment process was mainly performed on distributed points, where using check points as the evaluation criterion of result (Uysal et al., 2015). Nevertheless, the comparison between the aforementioned two methods has little been provided. This paper aims to evaluate the quality of DEMs obtained by UAV photogrammetry and contour interpolation through cross validation. In detail, two validation strategies with regards to point-based and area-based methods are used in this study.

\section{METHOD}

This paper presents a practical methods for efficient DEM construction from UAV images. The framework of the propose method is exhibited in Fig. 1. It contains three main components, namely (a) DEM generation through UAV photogrammetry, (b) DEM generation through contour interpolation, and (c) cross validation on quality of DEMs. The three components of the proposed method are described in detail in the following sections.

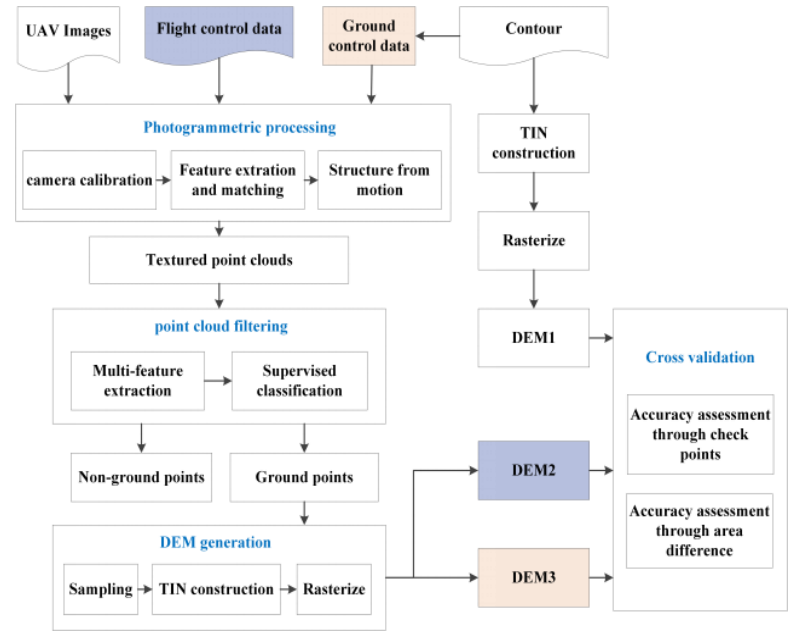

Figure 1.Framework of the proposed method 


\subsection{DEM Generation through UAV Photogrammetry}

We first use a structure-from-motion method to generate a sparse point clouds from UAV images (Torresani et al., 2008). In detail, a set of SIFT features is extracted from multi-view UAV images, followed by camera self-calibration through matching SIFT features (Ke and Sukthankar, 2004). With that, a small number of ground control points (GCPs) are used for bundle adjustment in order to define the correct mapping datum, leading to a set of sparse point cloud in the user's coordinate system (Xu et al., 2016). We then use the Patch-based Multiview (PMVS2) (Furukawa and Ponce, 2010) to obtain a dense set of point clouds with RGB color.

Next, we filter out the non-ground points from the UAVderived point cloud by using a supervised classification method. Specifically, a combined feature descriptor with consideration of spectral and geometrical features are extracted from the UAV image-derived point clouds. Based on the feature descriptor, the Support Vector Machine (SVM) (Cortes and Vapnik, 1995) classifier is used in an one-to-all configuration, for its considering its efficiency and extensive implementation.

We then sample the ground points with uniform interval, and construct triangular irregular networks (TIN) using the determination of earth surface structure (DEST) algorithm (Tarquini et al., 2007). Finally, TINs are converted to raster using the liner interpolation method, leading to UAV-DEM (Blu et al., 2004).

\subsection{DEM Generation through Contour Interpolation}

This step aims to generate DEM from digitized contours through ESRI ArcGIS ${ }^{\circledR}$ 10.0. To begin with, we transform the contour lines into a seamless TIN by mathematical calculations. Following that, the rasterization process is performed, result in a DEM, called as Contour-DEM in the rest of this paper.

\subsection{Cross Validation on Quality of DEMs}

We perform a cross validation on the quality of UAV-DEM and Contour-DEM from three aspects. First, we compare the spatial resolution of UAV-DEM and Contour-DEM. Second, we evaluate the accuracy of UAV-DEMs using a set of invariant check points from topographic map. In this process, UVA-DEMs georeferenced with both flight control data (POS) and ground control points (GCPs) are further compared. Third, we evaluate the quality of Contour-DEM on the basis of UAVDEM from the whole area instead of using individual points.

\section{STUDY AREA AND DATA ACQUISITION}

\subsection{Study Area}

The study area was in the east of Jilin province in China

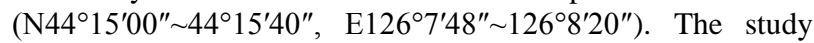
area was subordinated to the Songliao Plain, which was characterized by plane terrain and fertile soil. It mainly included two types of coverage with respect to lush vegetation and bare ground with coverage area of $0.32 \mathrm{~km}^{2}$ (Figure 2).

\subsection{Data acquisition}

3.2.1 UAV Imaging: A fixed-wing UAV was employed for image shooting. It was automatically controlled by a ground control station with a predefined trajectory at an average height of $600 \mathrm{~m}$ above the sea level. The flight control data of each flight were obtained by a low-cost flight control system. Overlap degrees in forward and side directions were measured with values of $90 \%$ and $70 \%$, respectively. A digital camera SONY DSC-WX220 was used to perform image shooting over the study area. In total of 157 images were collected with size of $4896 \times 3672$ pixels from eight parallel routes. The geometrical resolution, indicated by ground sampling distance (GSD) is about $2 \mathrm{~cm}$, which enables all the objects to be clearly identified.

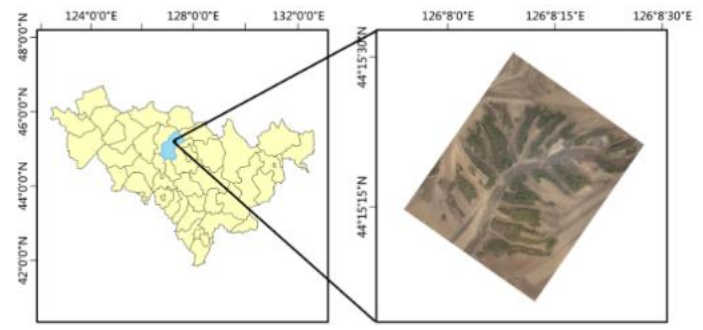

Figure 2. Study area in Jiutai, Jilin Province, China

\begin{tabular}{|c|c|c|c|}
\hline \multicolumn{2}{|c|}{ Parameters of UAV system } & \multicolumn{2}{c|}{ Parameters of camera } \\
\hline $\begin{array}{c}\text { Flight } \\
\text { altitude }\end{array}$ & $600 \mathrm{~m}$ & Model & $\begin{array}{c}\text { SONY DSC- } \\
\text { WX220 }\end{array}$ \\
\hline Overlap & $90 \% / 70 \%$ & Pixel size & $4896 \times 3672$ \\
\hline Flight lines & 8 & $\begin{array}{c}\text { Spatial } \\
\text { resolution }\end{array}$ & $2 \mathrm{~m}$ \\
\hline
\end{tabular}

Table 1.Specific parameters

3.2.2 Field Surveying: Topographic data was obtained through field measurements by GPS-RTK, leading to a maximum error of $1 \mathrm{~cm}$ for each points. In total of 24 identification points were chosen from 1:50000 contour (Fig. $3), 12$ of which were used as ground control points and the remaining points were used as check points.

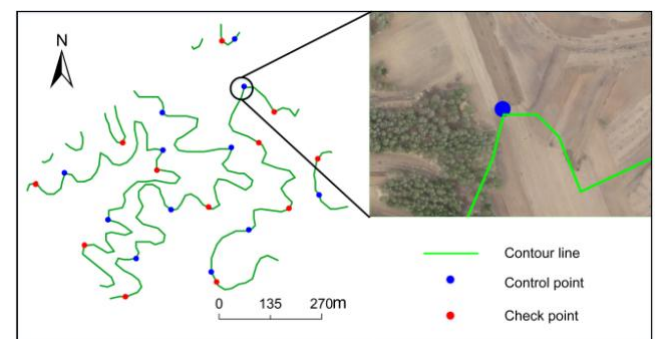

Figure 3. The map of identification points

\begin{tabular}{|c|c|c|c|c|c|c|c|}
\hline GCP & $\mathrm{X}$ & $\mathrm{Y}$ & $\mathrm{Z}$ & GCK & $\mathrm{X}$ & $\mathrm{Y}$ & $\mathrm{Z}$ \\
\hline 1 & 73 & 6058 & 240 & 1 & 6 & 6036 & 240 \\
\hline 2 & 298 & 6197 & 250 & 2 & 206 & 6128 & 250 \\
\hline 3 & 463 & 6366 & 250 & 3 & 438 & 6366 & 250 \\
\hline 4 & 301 & 6112 & 240 & 4 & 117 & 5890 & 240 \\
\hline 5 & 489 & 6258 & 260 & 5 & 285 & 6067 & 240 \\
\hline 6 & 174 & 5950 & 240 & 6 & 558 & 6201 & 260 \\
\hline 7 & 460 & 6115 & 250 & 7 & 215 & 5772 & 250 \\
\hline 8 & 238 & 5861 & 250 & 8 & 424 & 5805 & 260 \\
\hline 9 & 320 & 5972 & 250 & 9 & 593 & 5975 & 260 \\
\hline 10 & 412 & 5829 & 260 & 10 & 522 & 6129 & 260 \\
\hline 11 & 1301 & 5924 & 260 & 11 & 408 & 5978 & 250 \\
\hline 12 & 1463 & 6007 & 270 & 12 & 659 & 6090 & 270 \\
\hline
\end{tabular}


Table 2. Coordinate of the identification points

\section{RESULTS AND DISCUSSION}

Figs. 4(a-b) display the UAV-DEMs with resolution of 5m, georeferenced by POS data and GCPs, respectively. Fig. 4(c) displays the Contour-DEM with resolution of $5 \mathrm{~m}$. Note that, UAV-DEMs reveal more geographic information compared to Conture-DEM, reflecting the varied topographic changes. Specifically, UAV-DEMs clearly and vividly exhibit complete context of undulating topography, while Contour-DEM only reflects the overall trend of decreasing from southwest to northeast. Moreover, the regional elevation range of UAVDEMs in Figs. 4(a-b) are [227.245, 283.840] and [234.170, 269.574], respectively, whereas the elevation range of ContureDEM is $[240,270]$.

Fig. 5 shows the accuracy of UAV-DEMs, georeferenced by both POS data and GCPs, respectively. In view of the accuracy assessed through check points, the mean errors in z-coordinate of UAV-DEMs geoferenced with POS data and GCPs are $1.27 \mathrm{~m}$ and $-0.25 \mathrm{~m}$, respectively. The result of error distribution confirm that although the former is relatively discrete, applied GCPs contribute to a remarkable improvement of the overall accuracy, with a RMSE error of $\sim \pm 0.5 \mathrm{~m}$. From the above experimental result, we can conclude that the UAV-DEM meet the requirement of digital production and geographical application when a small number of GCPs are available.

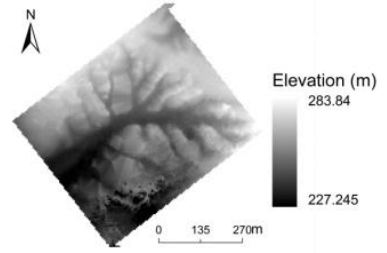

(a)

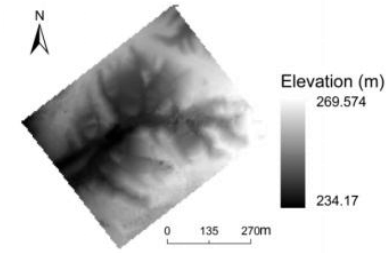

(b)

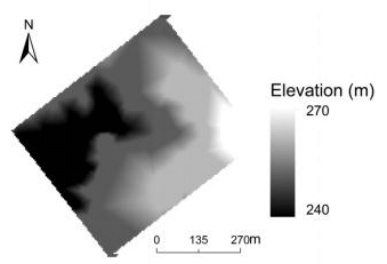

(c)

Figure 4. UAV-DEM georeferenced by (a) POS acquired by UAV, and by (b) GCPs, (c) Contour-DEM.

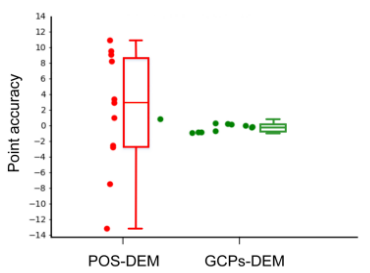

(a)

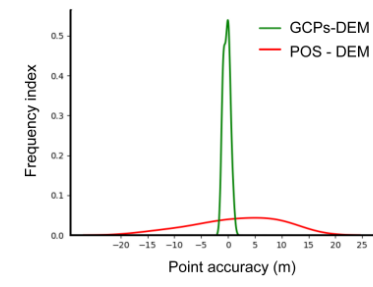

(b)
Figure 5. Accuracy of UAV-DEMs, georeferenced by POS and GCPs. (a) indicates the absolute error on the $\mathrm{Z}$ coordinate and (b) shows the error distribution

Figs. 6(a-b) shows the comparison between the Contour-DEM and UAV-DEMs, georeferenced by POS data and GCPs, respectively. The comparison results indicate that the terrain elevation cannot be completely obtained by linear interpolating the measurable contours, leading to significant errors in unmeasurable area. Fig. 6(a) shows a rotational distortion, which is resulted from varied flight control data. However, the difference in Fig. 6(b) were reduced by using evenly arranged control points and consequently improved the accuracy of the spatial elevation variation between Contour-DEM and UAVDEM. Namely, the conclusion of cross validation reflected the overall accuracy of DEM generation from contour through linear interpolation.

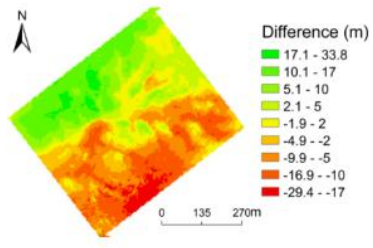

(a) (b)

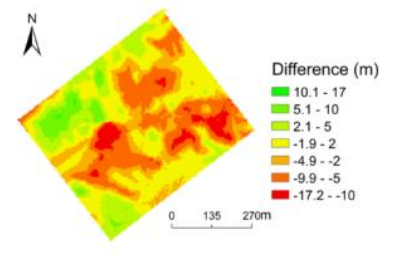

Figure 6. Comparison between Contour-DEM and UAV-DEMs, georeferenced by (a) POS and by (b) GCPs

In addition, Fig. 7. show the frequency histogram of difference values between Contour-DEM and UAV-DEMs, georeferenced by fight control data and ground control points, respectively. Two frequency histogram all nearly follow normal distribution, but the latter was more symmetric, which suggested the difference remain stable.

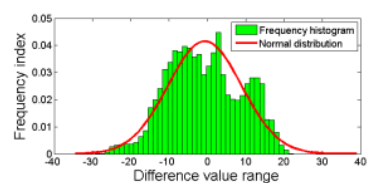

(a) (b)

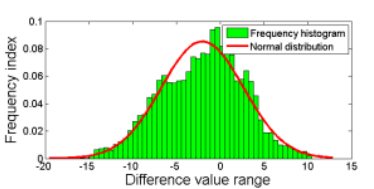

Figure 7. Frequency histogram of difference values between Contour-DEM and UAV-DEMs, georeferenced by (a) POS and (b) by GCPs

\section{CONCLUSIONS}

This article investigates the feasibility and practicality of UAV applied to DEM generation through advanced photogrammetric techniques. In addition, a number of GCPs considered during multi-vision matching processing has been proved to improve the accuracy of DEM generated from UAV images than using flight control data (POS). The better result shows the accuracy assessed reference check points has achieved the level in decimeter, which is acceptable for overwhelming majority geographical research and engineering production based on DEM. Conclusively, UAV-DEM is extremely alike to the true geo-digital elevation model. A comparison of Contour-DEM with UAV-DEM reveal that DEM generated from contour linear interpolation inevitably has elevation deviation due to conditional limitations of traditional field measurements. It proves the superiority of DEM construction using UAV iamges. Further studies may focus on simplifying the introduced framework for rapid and automatic UAV-DEM construction and optimizing algorithms so as to improve the accuracy of outcomes.

\section{ACKNOWLEDGEMENTS (OPTIONAL)}


This work is partially supported by grants from the National Natural Science Foundation of China (grant \# 41701534)

\section{REFERENCES}

Ardiansyah, P.O.D., and Yokoyama, R. (2002). DEM generation method from contour lines based on the steepest slope segment chain and a monotone interpolation function. ISPRS Journal of Photogrammetry and Remote Sensing 57, 86101.

Arun, P.V. (2013). A comparative analysis of different DEM interpolation methods. The Egyptian Journal of Remote Sensing and Space Science 16, 133-139.

Blu, T., Thévenaz, P., and Unser, M. (2004). Linear interpolation revitalized. IEEE Transactions on Image Processing 13, 710-719.

Cortes, C., and Vapnik, V. (1995). Support-vector networks. Machine learning 20, 273-297.

De Souza, C.H.W., Lamparelli, R.A.C., Rocha, J.V., and Magalhães, P.S.G. (2017). Height estimation of sugarcane using an unmanned aerial system (UAS) based on structure from motion (SfM) point clouds. International journal of remote sensing 38, 2218-2230.

Demirkesen, A.C. (2012). Multi-risk interpretation of natural hazards for settlements of the Hatay province in the east Mediterranean region, Turkey using SRTM DEM. Environmental Earth Sciences 65, 1895-1907.

Furukawa, Y., and Ponce, J. (2010). Accurate, dense, and robust multiview stereopsis. IEEE transactions on pattern analysis and machine intelligence $32,1362-1376$.

Jones, J.W., Desmond, G.B., Henkle, C., and Glover, R. (2012). An approach to regional wetland digital elevation model development using a differential global positioning system and a custom-built helicopter-based surveying system. International journal of remote sensing 33, 450-465.

Ke, Y., and Sukthankar, R. (2004). PCA-SIFT: A more distinctive representation for local image descriptors. Paper presented at: Computer Vision and Pattern Recognition, 2004 CVPR 2004 Proceedings of the 2004 IEEE Computer Society Conference on (IEEE).
Liang, W., Bai, D., Wang, F., Fu, B., Yan, J., Wang, S., Yang, Y., Long, D., and Feng, M. (2015). Quantifying the impacts of climate change and ecological restoration on streamflow changes based on a Budyko hydrological model in China's Loess Plateau. Water Resources Research 51, 6500-6519.

Maxwell, A.E., and Warner, T.A. (2015). Differentiating minereclaimed grasslands from spectrally similar land cover using terrain variables and object-based machine learning classification. International Journal of Remote Sensing 36, 4384-4410.

Mukherjee, S., Joshi, P., Mukherjee, S., Ghosh, A., Garg, R., and Mukhopadhyay, A. (2013). Evaluation of vertical accuracy of open source Digital Elevation Model (DEM). International Journal of Applied Earth Observation and Geoinformation 21, 205-217.

Rao, E., Ouyang, Z., Yu, X., and Xiao, Y. (2014). Spatial patterns and impacts of soil conservation service in China. Geomorphology 207, 64-70.

Rock, G., Ries, J., and Udelhoven, T. (2011). Sensitivity analysis of UAV-photogrammetry for creating digital elevation models (DEM). Paper presented at: Proceedings of Conference on Unmanned Aerial Vehicle in Geomatics.

Shi, P., and Yu, D. (2014). Assessing urban environmental resources and services of Shenzhen, China: A landscape-based approach for urban planning and sustainability. Landscape and Urban Planning 125, 290-297.

Tarquini, S., Isola, I., Favalli, M., Mazzarini, F., Bisson, M., Pareschi, M.T., and Boschi, E. (2007). TINITALY/01: a new triangular irregular network of Italy. Annals of Geophysics.

Torresani, L., Hertzmann, A., and Bregler, C. (2008). Nonrigid structure-from-motion: estimating shape and motion with hierarchical Priors. IEEE Transactions on Pattern Analysis \& Machine Intelligence 30, 878-892.

Uysal, M., Toprak, A., and Polat, N. (2015). DEM generation with UAV Photogrammetry and accuracy analysis in Sahitler hill. Measurement 73, 539-543.

Xu, Z., Wu, L., Gerke, M., Wang, R., and Yang, H. (2016). Skeletal camera network embedded structure-from-motion for 3D scene reconstruction from UAV images. ISPRS Journal of Photogrammetry and Remote Sensing 121, 113-127.

Revised March 2018 